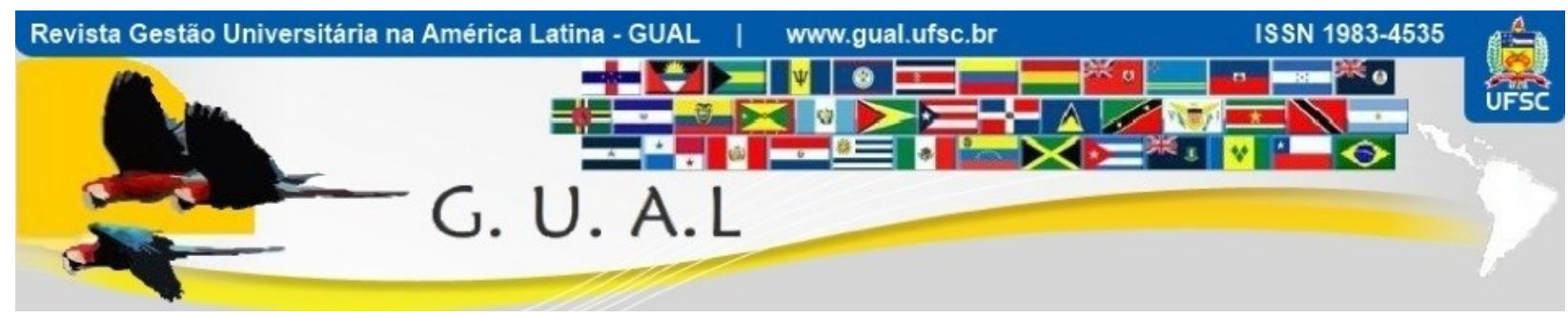

DOI: http://dx.doi.org/10.5007/1983-4535.2014v7n2p130

\title{
ANÁLISE ENVOLTÓRIA DE DADOS (DEA): UMA PROPOSTA PARA AVALIAÇÃO DE DESEMPENHO DE UNIDADES ACADÊMICAS DE UMA UNIVERSIDADE
}

\section{DATA ENVELOPMENT ANAL YSIS (DEA): A PROPOSAL FOR PERFORMANCE EVALUATION OF UNIVERSITY ACADEMIC UNITS}

Cintia Paese Giacomello, Doutora Universidade de Caxias do Sul - UCS cpaese1@ucs.br

Ronald Lopes de Oliveira, Mestre Universidade de Caxias do Sul - UCS rlolive2@ucs.br

Recebido em 17/outubro/2013

Aprovado em 27/abril/2013

Sistema de Avaliação: Double Blind Review

Esta obra está sob uma Licença Creative Commons Atribuição-Uso. 


\title{
RESUMO
}

Este artigo apresenta uma aplicação da Análise Envoltória de Dados para avaliar unidades de ensino da Universidade de Caxias do Sul. Esta técnica analisa a produtividade (ou eficiência) de Unidades Tomadoras de Decisão através das melhores práticas, sugerindo uma classificação e indicações de variáveis que devam ser melhoradas. Foram utilizadas sete variáveis de caráter financeiro, sendo quatro de entrada e três de saída para 20 unidades. Os resultados permitiram classificar as unidades pelo grau de eficiência, formando três grupos. Para o grupo das unidades de baixa eficiência foram apresentados os valores que cada variável deveria alcançar, bem como as unidades eficientes que poderiam servir de benchmark.

Palavras-chave: Gestão universitária. Avaliação de desempenho. Análise Envoltória de Dados.

\begin{abstract}
This paper presents an application of Data Envelopment Analysis to evaluate university units. The technique analyzes the productivity (or efficiency) of Decision Making Units based on best practices. DEA results indicate variables classifications and points that must be improved. We have applied it in seven financial variables, including four input and three output variables to 20 university units. Results have allowed us to classify them in three groups by degree of efficiency. Units group with low efficiency have presented values that each variable should achieve, as well as colleges efficient that may serve as a benchmark.
\end{abstract}

Keywords: University management. Performance Evaluation. Data Envelopment Analysis. 


\section{INTRODUÇÃO}

A proliferação de instituições de ensino superior a partir da década de 90 no Brasil gerou um crescente número de ofertas de cursos de graduação. No ano de 2010 foram oferecidos 29.507 cursos em todo o território nacional (INEP, 2010), apresentando um crescimento de $102,25 \%$ entre os anos de 2001 e 2010 . Na esfera das instituições privadas este crescimento é mais expressivo: enquanto a quantidade de alunos nos cursos presenciais de instituições públicas cresceu $64,8 \%$, nas privadas o crescimento, no mesmo período, foi de $120,6 \%$.

Se por um lado o MEC busca maneiras de avaliar estes cursos para garantir o mínimo de qualidade oferecida aos alunos, por outro lado as instituições de ensino superior se veem cada vez mais forçadas a sistematicamente oferecerem novos "produtos" para atraírem alunos e manterem sua posição e espaço no mercado.

Tal concorrência, aliada às dificuldades internas que algumas instituições privadas enfrentam em função de orçamentos limitados acaba gerando uma disputa pelos poucos recursos disponíveis para investimentos. O estudo realizado por Kyj e Parker (2008) apresentou uma investigação sobre os fatores que precedem a prática da participação orçamentária nas organizações. Os autores afirmaram que, efetivamente, muitas pesquisas tratam apenas sob o aspecto comportamental dos empregados. O que os autores evidenciam é que existem condições que impulsionam a participação orçamentária, como por exemplo; o estilo de liderança e a relação intersetorial na IES. Desta forma, em uma instituição que possui setores de diferentes áreas do conhecimento exige que estas premissas da participação orçamentária sejam observadas.

Assim, os gestores das IES deparam-se diariamente com dúvidas do tipo: como está o desempenho dos cursos Graduação? Onde é prioritário investirmos os recursos? Quais aspectos devem ser melhorados neste curso? O que pode ser utilizado de um curso como exemplo para outros cursos? Para responder estas dúvidas parte-se da questão de origem que é: como avaliar a eficiência dos cursos oferecidos? Como avaliar o desempenho da unidade acadêmica levando em consideração seus produtos?

A complexidade muitas vezes encontrada para responder as questões citadas acima, exige que esforços sejam lançados para garantir a qualidade e sustentabilidade dos produtos ofertados pelas IES. Ferramentas que permitam medir de forma adequada o desempenho dos produtos e consequentemente das unidades acadêmicas surgem como elementos promotores 
do sucesso das instituições. Segundo Kaplan e Norton (2001), a estratégia é fundamental para uma organização, mas a execução com êxito da estratégia é o principal problema das organizações, ou seja, de acordo com o autor, as instituições falham na execução dos planos e seus mecanismos de monitoramento. Neste sentido, Berliner e Brimson (1992), afirmam que a utilização de instrumentos de medição de desempenho é fundamental para garantir a execução do planejamento estratégico da empresa.

Hammer (2007) cita que nos últimos anos as organizações desenvolveram sofisticados sistemas de medição da estratégia, baseados em ferramentas como o Balanced Scorecard (BSC), Key Performance Indicators (KPYs), entre outras, mas é primordial identificar os fatores críticos para o sucesso dos negócios e identificar os indicadores para expressá-los. Apesar de ser possível encontrar na literatura vasto material sobre o tema Avaliação de Desempenho, o conjunto de técnicas orientadas para o tratamento de indicadores, segundo diversas perspectivas, valendo-se de metodologias de análise multicriterial ainda apresenta uma lacuna importante a ser explorada.

Neste sentido, uma das maneiras possíveis de fazer esta análise e medição é através da Análise Envoltória de Dados (DEA). Esta técnica, largamente utilizada na área da Engenharia de Produção e Contabilidade, consiste em uma técnica multivariada não paramétrica que analisa a produtividade (ou eficiência) de Unidades Tomadoras de Decisão (Decision Making Units - DMUs), com base nas melhores práticas. Através da comparação entre as unidades, ela fornece dados quantitativos sobre possíveis direções para melhorar o desempenho das unidades ineficientes.

Para tanto, o conjunto das unidades deve ser homogêneo e possuir as mesmas informações de entrada (inputs) e de saída (outputs). Como produto da Análise Envoltória de Dados tem-se a curva de eficiência (ou de máxima produtividade), considerando a relação ótima entrada/saída.

A Análise Envoltória de Dados teve sua origem na década de 70, com os primeiros trabalhos sendo originados no trabalho de Charnes, Cooper e Rhodes de 1978. Ela já foi utilizada em diversos setores, desde avaliação de bancos, companhias aéreas e organizações.

Como alguns exemplos de utilização da DEA em serviços no Brasil pode-se citar os trabalhos de Encinas (2010) e Vilela et al. (2007), respectivamente nas auditorias operacionais do Tribunal de Contas da União e em dados de cooperativas de crédito rural. Faria, Jannuzzi e Silva (2008) analisaram a eficiência dos gastos municipais da cidade do Rio 
de Janeiro em saúde e educação. Antoun Netto e Lins (2012) aplicaram a DEA para determinação de indicadores de desempenho na área da saúde das capitais federais. Calvo (2002), Marinho (2003), Lins et al. (2007) e Lobo (2010) realizaram estudos utilizando a análise envoltória de dados (DEA) voltados para hospitais, como uma unidade prestadora de serviços, com suas características de propriedade, porte, especialização, gestão, equipe de profissional, estrutura administrativa, públicos, privados, sistema de reembolso por serviços e o escopo de serviços.

$\mathrm{Na}$ área acadêmica, a aplicação da DEA também é expressiva. Recentemente Oliveira et al. (2011) realizaram um levantamento bibliográfico dos modelos DEA aplicados no setor educacional no Brasil no período de 1999 a 2009, onde através da pesquisa encontraram 71 (setenta e um) trabalhos realizados com a utilização do modelo Análise Envoltória de Dados.

A vantagem do uso desta técnica consiste no fato dela utilizar a eficiência relativa, sem prejuízo às unidades de pequeno porte. Ainda, mais de uma unidade pode ser classificada como eficiente, compondo a fronteira de eficiência relativa e servindo como referência para a atuação das demais unidades. Para as unidades ineficientes, são apresentadas contribuições de melhoria, com o estabelecimento de metas de atuação, e neste sentido, os fatores que contribuiriam para seu baixo desempenho podem ser desmembrados sugerindo pontos específicos de atuação.

Assim, propõe-se através deste trabalho construir um modelo de avaliação da eficiência das unidades acadêmicas com seus respectivos produtos (cursos) na Universidade de Caxias do Sul, através da utilização da Análise Envoltória de Dados. As principais etapas a serem seguidas para atingir os objetivos deste trabalho são:

a) identificar um conjunto de indicadores de entrada;

b) identificar um conjunto de indicadores de saída;

c) propor o modelo de avaliação;

d) classificar as unidades segundo a eficiência apresentada;

e) propor um sistema de alocação de recursos, em função da eficiência;

f) identificar os pontos que devem ser melhorados nas unidades ineficientes.

A Universidade de Caxias do Sul é uma instituição de ensino superior, mantida pela Fundação Universidade de Caxias do Sul (FUCS), entidade jurídica de direito privado, sem fins lucrativos de caráter comunitário e regional, com atuação direta na região nordeste do 
estado do Rio Grande do Sul. Dos seus Conselhos (Diretor e Curador) participam representantes dos municípios abrangidos pela Universidade, entidades da comunidade e representantes dos governos, em nível federal, estadual e municipal. Mantém unidades em nove cidades, atingindo diretamente uma população de mais de 1 milhão de habitantes. Seu Campus Sede está localizado na cidade de Caxias do Sul. Foi fundada em 1967 e, ao longo de sua existência já graduou mais de 100 mil pessoas. Atualmente, possui em torno de 36 mil alunos e 1,1 mil professores em 80 cursos de graduação, 70 Cursos de Especialização e 13 Programas de Pós-Graduação Stricto Sensu com 14 Mestrados e 4 Doutorados.

\section{AVALIAÇÃO DE DESEMPENHO EM INSTITUIÇÕES DE ENSINO}

A sobrevivência das instituições de ensino superior é diretamente afetada por fatores internos e externos, independente das características e particularidades de cada IES. Neste aspecto, não diferente de outras organizações, as IES também necessitam cumprir metas que visem o alcance de objetivos da própria instituição e, especialmente, que atendam às regulações e exigências legais fixadas pelo governo. Desta forma, a criação de objetivos estratégicos estimula a organização para a busca de melhores resultados, e é necessário fixar medidas de desempenho para avaliar os resultados alcançados. A melhoria do desempenho organizacional só é possível quando se consegue medir diferentes dimensões ou perspectivas.

Segundo Mañas (1993), os executivos devem buscar uma ação coordenada que proporcione o aumento da competitividade, incentivando inovações e melhorias no ambiente organizacional, além da busca de novas oportunidades para que a empresa conquiste vantagens competitivas diante dos concorrentes.

De acordo com Smith (2005), gerenciamento do desempenho é a estratégia e o processo para alavancar as melhores práticas, metodologias e tecnologias para gerir o desempenho de uma organização e suas redes de negócios, utilizando seus ativos para alcançar um conjunto comum de metas e objetivos. O gerenciamento do desempenho foca na compreensão, otimização e em ações e decisões de alinhamento, além de buscar garantir a colaboração de todos os indivíduos envolvidos com o negócio. Desta forma, permite-se alinhar ações nos níveis estratégico, tático e operacional, de modo a garantir um resultado ótimo.

Os principais objetivos de um sistema de medição de desempenho são: 
a) apresentar um quadro equilibrado dos diferentes aspectos de desempenho;

b) garantir um ambiente consistente e uma sistemática de medição de desempenho;

c) apresentar as informações de forma rápida, com fácil interpretação por todos os segmentos da organização.

A premissa básica para qualquer sistema de medição de desempenho é orientar a melhoria contínua e o desempenho operacional. Neste sentido, Smith (2005), afirma que o gerenciamento do desempenho provê um fundamento para as organizações gerirem os indivíduos para fazê-los tomar as decisões certas de modo a maximizar a eficiência.

O monitoramento do desempenho nos diversos níveis organizacionais e sua aderência aos interesses estratégicos das organizações costuma ser realizado a partir de um conjunto de indicadores de desempenho, que tornam-se instrumentos de apoio à implantação da estratégia organizacional.

Levando em consideração as citações acima, pode-se afirmar que a avaliação de desempenho é mais que uma ferramenta gerencial: é uma medida estratégica de sobrevivência das organizações, sendo assim, diversos modelos têm sido utilizados pelos diversos setores, como também por instituições de ensino superior.

Segundo Macedo (2004), não existe nenhum método ou modelo de avaliação de desempenho organizacional que seja único para toda e qualquer variável do mundo empresarial. Em vez disso, os gestores e analistas se utilizam de uma série de metodologias de avaliação de desempenho para lidar com os diferentes elementos de uma organização.

Nos últimos anos diversas metodologias de avaliação de processos de desempenho foram utilizadas pelas organizações, como por exemplo:

a) TOC - Theory of Constrains (Teoria das Restrições) proposta por Goldratt e Fox (1992);

b) TQM - Total Quality Management (Gerenciamento pela Qualidade Total): ver Campos (1993);

c) BSC - Balanced Scorecard, por Kaplan e Norton (2001).

Estas técnicas utilizadas individualmente ou em conjunto contribuem para a avaliação das organizações, bem como, permitem a melhora do desempenho das unidades avaliadas. Entretanto, a variedade e diversidade de fatores a fim de analisar e resolver problemas de 
maior complexidade abriu espaço para que a análise multicriterial pudesse ser utilizada como apoio na comparação de desempenho.

Segundo Saaty (1991), a análise multicriterial torna mais claro quais são as vantagens e desvantagens de cada alternativa possível, sob condições de risco e incerteza, servindo também como ferramenta para formulação de estratégias corporativas necessárias à competitividade.

Neste contexto, foi utilizada a técnica de Análise Envoltória de Dados (DEA), que permite avaliar unidades tomadoras de decisão e identificar resultados eficientes entre uma lista de possíveis candidatos.

Através da técnica DEA é possível verificar se cada unidade opera de maneira adequada ou não, relativamente a um elenco específico de recursos utilizados e de resultados obtidos, em comparação com unidades consideradas similares por seus administradores, sem a necessidade de conhecer a priori qualquer relação de importância (pesos) entre as variáveis consideradas. Desta maneira, esta técnica não suplementa somente métodos de avaliação de desempenho, mas também possibilita uma melhor visualização de como a organização e suas unidades tomadoras de decisão estão realmente se comportando.

A técnica DEA tem sido utilizada na avaliação de unidades de ensino desde o seu início, na década de 1970 com a avaliação de trabalhos escolares no estado do Texas - EUA por Charnes, Cooper e Rhodes (1978).

A partir deste ponto, outros trabalhos foram realizados em instituições de ensino. No Brasil, podem ser citados alguns trabalhos. Cavalcante e Andriola (2012) analisaram os cursos de graduação da Universidade Federal do Ceará. Casado e Siluk (2011) avaliaram a eficiência de nove unidades de uma mesma universidade com três modelos: um geral, um para a graduação e outro para pós-graduação. Costa (2010) avaliou 49 instituições públicas federais.

Casado e Souza (2007) fizeram uma análise do uso da DEA no ensino superior, citando 1995 como o ano inicial das aplicações neste setor. Oliveira e Turrioni (2006) avaliaram 19 instituições federais de ensino superior. Gomes et al. (2007) identificaram formas de alocação de bolsas de iniciação científica na EMBRAPA. Lins et al. (2004) avaliaram o desempenho da pós-graduação. Panepucci (2003) avaliou o desempenho dos departamentos acadêmicos da UFSCar. Façanha e Marinho (2001) avaliaram as diferenças no desempenho de instituições de ensino superior em diferentes regiões brasileiras. Belloni (2001) avaliou 33 universidades federais brasileiras. 


\section{A METODOLOGIA dA ANÁLISE ENVOLTÓRIA DE DADOS}

A técnica DEA se baseia no pressuposto de que, se uma unidade tomadora de decisão (DMU) é capaz de produzir uma determinada "saída" Y (output) utilizando X entradas (inputs), então outras unidades também poderiam fazer o mesmo, caso elas estivessem atuando eficientemente.

Pode-se entender que a produtividade é calculada pela relação entre as saídas obtidas com uma certa quantidade de entradas. A eficiência, por sua vez, é avaliada pela comparação entre a produtividade observada e a produtividade máxima que poderia ser alcançada. Assim, a DEA se baseia nos princípios da eficiência.

Soares de Mello et al. (2005) apresentam um tutorial sobre DEA e trabalham os conceitos de produtividade e eficiência afirmando que eficiência é um conceito relativo que compara o que foi produzido, dados os recursos disponíveis, com o que poderia ter sido produzido com os mesmos recursos.

De forma geral, os objetivos da DEA são comparar um conjunto de unidades que realizam tarefas similares e se diferenciam quanto aos recursos que consomem e aos resultados que produzem.

Os principais objetivos da DEA, conforme Gomes et al. (2001) são:

a) comparar um certo número de DMUs que realizam tarefas similares e se diferenciam nas quantidades de inputs que consomem e de outputs que produzem;

b) identificar as DMUs eficientes, medir e localizar a ineficiência e estimar uma função de produção linear por partes (piecewise linear frontier), que fornece o benchmark para as DMUs ineficientes. Ao identificar as origens e quantidades de ineficiência relativas de cada uma das DMUs, é possível analisar qualquer de suas dimensões relativas a entradas e/ou saídas. A fronteira de eficiência compreende o conjunto de DMUs Pareto eficientes;

c) determinar a eficiência relativa das DMUs, contemplando cada uma,relativamente a todas as outras que compõem o grupo a ser estudado.Assim, sob determinadas condições, DEA pode ser usado na problemática da ordenação como ferramenta multicrédito de apoio à decisão;

d) subsidiar estratégias de produção que maximizem a eficiência das DMUs avaliadas, corrigindo as ineficientes através da determinação de alvos;

e) estabelecer taxas de substituição entre as entradas, entre as saídas e entre entradas e saídas, permitindo a tomada de decisões gerenciais;

f) considerar a possibilidade de os outliers não representarem apenas desvios em relação ao comportamento "médio", mas possíveis benchmarks a serem analisados pelas 
demais DMUs. Os outliers podem representar as melhores práticas dentro do universo investigado.

Os modelos de DEA podem ser orientados de duas formas: input ou output. Os modelos orientados a input visam obter um menor uso de inputs dados os níveis de outputs, ou seja, quando a eficiência é atingida por uma redução equiproporcional de entradas, mantidas as saídas constantes. Já os modelos orientados a outputs buscam obter o máximo nível de outputs mantendo os inputs fixos, então se deseja maximizar os resultados sem diminuir os recursos.

Além disso, há outra classificação de modelos, do ponto de vista dos modelos matemáticos utilizados nos cálculos: os modelos CCR e BCC.

O modelo CCR trabalha com retornos constantes de escala, ou seja, qualquer variação nas entradas (inputs) produz variação proporcional nas saídas (outputs). Ele foi proposto originalmente por Charnes, Cooper e Rhodes em 1978 e é também conhecido como CRS Constant Returns to Scale.

O modelo BCC, proposto por Banker, Charnes e Cooper em 1984, considera retornos variáveis de escala, por isso também é conhecido como VRS - Variable Returns to Scale. Ele substitui o axioma da proporcionalidade entre inputs e outputs pelo axioma da convexidade.

Assim os modelos básicos existentes são CCR - insumo orientado, CCR - produto orientado, BCC - insumo orientado e BCC - produto orientado. Esses quatro modelos estão descritos detalhadamente em Fried et al. (1993) e Charnes et al. (1994). As demonstrações matemáticas destes modelos também podem ser obtidas em Soares de Mello et al. (2005) no trabalho intitulado "Curso de Análise de Envoltória de Dados" apresentado no Simpósio Brasileiro de Pesquisa Operacional.

Uma vez analisado um conjunto de dados pela técnica DEA é possível calcular a eficiência, a eficiência invertida, a eficiência composta e a eficiência composta normalizada.

A eficiência é dada comparação entre a produtividade observada e a produtividade máxima que poderia ser alcançada. Ela é calculada pela razão entre a soma ponderada dos produtos pela soma ponderada dos insumos.

A eficiência invertida é uma forma de medir a ineficiência de uma DMU (ENTANI et al., 2002; NOVAES, 2002). A partir da interpretação e da proposta de Leta et al. (2005) a fronteira invertida permite a identificação de unidades produtivas consideradas "falsas eficientes", pois já que a equação da eficiência invertida propõe a divisão da soma ponderada 
dos insumos pela soma ponderada dos produtos (o inverso da fronteira clássica), passa a indicar como mais eficientes (inversamente) aquelas unidades que gastaram mais insumos e geraram menos produtos, enquanto a fronteira clássica classifica como melhor o que gerou mais produtos gastando menos insumo. Esta fronteira invertida é composta pelas DMUs com as piores práticas gerenciais (e pode ser chamada de fronteira ineficiente). Pode-se também afirmar que as DMUs pertencentes à fronteira invertida têm as melhores práticas sob uma ótica oposta (SOARES DE MELLO et al, 2005). Assim, ao avaliar a eficiência invertida, quanto mais baixo o valor, melhor se comporta a DMU.

Já a eficiência composta, descrita em Angulo Meza et al. (2005), é uma combinação da eficiência clássica e da invertida. Ela é obtida pela equação:

$$
\text { Eficiência composta }=\frac{\text { Eficiência clássica }+(1-\text { Eficiência invertida })}{2}
$$

A eficiência composta normalizada (apresentada como Composta*) é obtida dividindo o valor da eficiência composta pelo maior valor entre todos os valores de eficiência composta, conforme a equação:

$$
\text { Eficiência composta } *=\frac{\text { Eficiência composta }}{\text { Máximo (Eficiência Composta) }}
$$

Além das eficiências, é possível fazer análise dos benchmarks, ou parceiros de excelência, para orientar as unidades ineficientes. A força desta influência é dada por um coeficiente, o Índice de Importância de Referência, que varia entre zero e um. Quanto mais próximo de 1, mais forte é a influência que uma unidade exerce sobre a outra. A seguir são apresentadas as etapas desenvolvidas e resultados.

\section{METODOLOGIA}

Para a realização do estudo foram analisadas informações de 20 unidades acadêmicas da Universidade de Caxias do Sul. A opção de escolha de $\operatorname{todas}^{1}$ as unidades acadêmicas foi possível, devido ao modelo proposto utilizar somente variáveis que compõem insumos e

${ }^{1}$ A UCS possui atualmente 21 unidades universitárias, porém uma delas conta contava com 12 alunos matriculados em 2012 e foi excluída da análise. 
produtos relacionados ao ensino de graduação, ou seja, executam os mesmos processos, com objetivos semelhantes, variando somente a intensidade ou magnitude dos inputs e outputs para medir a eficiência.

O conceito de eficiência está relacionado com a utilização e alocação dos recursos. Dessa forma, para se obter valores confiáveis no cálculo de eficiência é necessário empregar indicadores que representem de forma consistente as características inerentes ao ensino de graduação da instituição.

A partir dos principais inputs e outputs utilizados por diversos trabalhos nas últimas décadas, do conjunto de indicadores de eficiência fornecidos pelas IES, e considerando a realidade da instituição em estudo, as variáveis a serem empregadas para a mensuração de eficiência das unidades acadêmicas neste trabalho encontram-se elencadas no Quadro 1.

Foi utilizado o programa SIADv.3.0 - Sistema Integrado de Apoio à Decisão, desenvolvido e detalhado em Angulo Meza et al. (2005) para realização das análises.

Para o desenvolvimento da aplicação do DEA foram seguidos os seguintes passos: seleção das variáveis de entrada e saída, coleta de dados das variáveis para as unidades acadêmicas estudadas (as DMU's), construção do modelo, processamento dos dados e análise dos resultados.

Foi utilizado o modelo CCR/output que é o mais apropriado neste caso, uma vez que busca maximizar os resultados, dados os insumos de cada Unidade. Assim, focou-se nos indicadores de eficiência orientados para a produção de resultados. A escolha do modelo CCR ao invés de BCC justifica-se também por este último atribuir eficiência $100 \%$ para unidades com menor input e maior output, independentemente da relação entre eles. Nesse caso, uma unidade poderia ser considerada eficiente só porque é de porte muito grande ou muito pequeno (SOARES de MELLO et al, 2004).

\begin{tabular}{|l|l|l|}
\hline Categoria & \multicolumn{1}{|c|}{ Variável } & \multicolumn{1}{c|}{ Descrição } \\
\hline \multirow{7}{*}{ Input } & Qde_cursos & Quantidade de cursos de graduação oferecidos pela DMU \\
\cline { 2 - 3 } & Custo_docente & Custo docente da DMU em atividades da graduação \\
\cline { 2 - 3 } & Cred_of & Quantidade de créditos oferecidos pela DMU \\
\cline { 2 - 3 } & Outras_desp & $\begin{array}{l}\text { Tudo que foi gasto nos cursos que não se caracteriza como hora } \\
\text { de professor em ensino de graduação. Todos os gastos gerais: } \\
\text { bolsas, depreciação, materiais, gastos gerais, viagens, estadias, } \\
\text { custos de eventos, etc... }\end{array}$ \\
\hline
\end{tabular}




\begin{tabular}{|l|l|l|}
\hline & & \\
\hline \multirow{3}{*}{ Output } & Qde_alunos & Quantidade de alunos na graduação \\
\cline { 2 - 3 } & Cred_ocup & Quantidade de créditos contratados pelos alunos \\
\cline { 2 - 3 } & Rec_cursos & Receita dos cursos \\
\hline
\end{tabular}

Quadro 1 Variáveis utilizadas no modelo Fonte: elaborado pelos autores.

Foram construídos dois modelos idênticos, um com dados do primeiro semestre de 2012 e outro com dados do segundo semestre. Optou-se por utilizar dois semestres, pois já foi constatado que historicamente há comportamentos diferentes entre eles. Os nomes das unidades foram codificados em respeito aos envolvidos.

\section{RESULTADOS E CONSIDERAÇÕES}

A Tabela 1 apresenta os resultados obtidos para os dois semestres, sendo as informações ordenadas de forma decrescente da média entre as eficiências compostas normalizadas dos dois semestres.

Os resultados apresentados na Tabela 1 indicam que a única unidade com eficiência $100 \%$ é a Unid 1, indicando ser a mais produtiva. Todas as demais unidades apresentam valores de eficiência composta normalizada inferiores a 1 .

Sabendo que quanto maior o valor da eficiência (exceto eficiência invertida) maior será a capacidade da unidade em obter os resultados a partir daqueles insumos, pode-se avaliar que somente Unid 1 obteve eficiência 100\%. As demais, para chegarem à eficiência, teriam que aumentar todos os seus produtos, mantendo fixos os mesmos recursos.

O comparativo entre o primeiro e o segundo semestres indica que houve mudança significativa na eficiência composta normalizada para duas unidades acadêmicas: Unid 11 e Unid 15. No primeiro semestre a Unid 11 apresentou eficiência composta normalizada de 0,7962, ocupando a nona posição entre as 20 unidades. Já no segundo semestre sua eficiência composta normalizada foi de 0,6668 , indicando posição $15^{\text {a }}$ entre as 20 . Isso indica uma piora relativa às demais unidades no segundo semestre. Da mesma forma a Unid 15 ocupava a posição $12^{\mathrm{a}}$ no primeiro semestre, com eficiência composta normalizada de 0,7435 e passou para a $16^{\mathrm{a}}$ posição no segundo semestre com eficiência composta normalizada de 0,6449 . 
Tabela 1 Medidas de Eficiência padrão, invertida e composta

\begin{tabular}{|c|c|c|c|c|c|c|c|c|c|}
\hline & \multicolumn{4}{|c|}{ Primeiro semestre } & \multicolumn{4}{|c|}{ Segundo semestre } & \multirow[b]{2}{*}{ 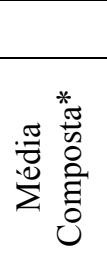 } \\
\hline & 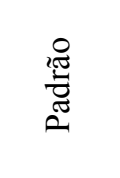 & 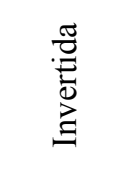 & $\begin{array}{l}\mathbb{\Xi} \\
0 \\
0 \\
0 \\
0 \\
0 \\
0\end{array}$ & $\begin{array}{l}\text { * } \\
\stackrel{\Xi}{0} \\
0 \\
0 \\
0 \\
0\end{array}$ & 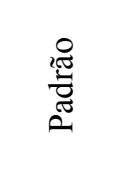 & 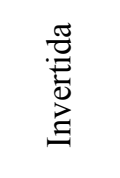 & 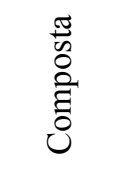 & 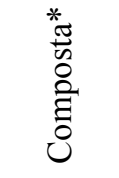 & \\
\hline Unid 1 & 1,0000 & 0,7003 & 0,6498 & 1,0000 & 1,0000 & 0,6738 & 0,6631 & 1,0000 & 1,0000 \\
\hline Unid 2 & 1,0000 & 0,7156 & 0,6422 & 0,9883 & 1,0000 & 0,6918 & 0,6541 & 0,9865 & 0,9874 \\
\hline Unid 3 & 1,0000 & 0,7470 & 0,6265 & 0,9641 & 1,0000 & 0,7304 & 0,6348 & 0,9573 & 0,9607 \\
\hline Unid 4 & 1,0000 & 0,8298 & 0,5851 & 0,9004 & 1,0000 & 0,8138 & 0,5931 & 0,8945 & 0,8974 \\
\hline Unid 5 & 1,0000 & 0,8375 & 0,5812 & 0,8944 & 1,0000 & 0,8103 & 0,5948 & 0,8971 & 0,8958 \\
\hline Unid 6 & 1,0000 & 0,8662 & 0,5669 & 0,8724 & 1,0000 & 0,8273 & 0,5863 & 0,8843 & 0,8783 \\
\hline Unid 7 & 1,0000 & 0,9156 & 0,5422 & 0,8343 & 1,0000 & 0,9099 & 0,5451 & 0,8220 & 0,8282 \\
\hline Unid 8 & 1,0000 & 0,9363 & 0,5319 & 0,8184 & 0,9648 & 0,9411 & 0,5118 & 0,7719 & 0,7952 \\
\hline Unid 9 & 1,0000 & 1,0000 & 0,5000 & 0,7694 & 1,0000 & 1,0000 & 0,5000 & 0,7541 & 0,7617 \\
\hline Unid 10 & 1,0000 & 1,0000 & 0,5000 & 0,7694 & 1,0000 & 1,0000 & 0,5000 & 0,7541 & 0,7617 \\
\hline Unid 11 & 0,9193 & 0,8844 & 0,5174 & 0,7962 & 0,8674 & 0,9831 & 0,4422 & 0,6668 & 0,7315 \\
\hline Unid 12 & 0,9555 & 1,0000 & 0,4777 & 0,7352 & 0,9449 & 0,9887 & 0,4781 & 0,7210 & 0,7281 \\
\hline Unid 13 & 0,8548 & 0,9396 & 0,4576 & 0,7042 & 0,8624 & 0,8877 & 0,4873 & 0,7350 & 0,7196 \\
\hline Unid 14 & 0,8866 & 0,9769 & 0,4549 & 0,6999 & 0,9063 & 0,9336 & 0,4863 & 0,7335 & 0,7167 \\
\hline Unid 15 & 0,8883 & 0,9220 & 0,4831 & 0,7435 & 0,8553 & 1,0000 & 0,4276 & 0,6449 & 0,6942 \\
\hline Unid 16 & 0,8689 & 1,0000 & 0,4344 & 0,6685 & 0,9272 & 1,0000 & 0,4636 & 0,6992 & 0,6839 \\
\hline Unid 17 & 0,8615 & 1,0000 & 0,4307 & 0,6628 & 0,7451 & 1,0000 & 0,3726 & 0,5619 & 0,6123 \\
\hline Unid 18 & 0,7393 & 0,9784 & 0,3805 & 0,5855 & 0,7450 & 1,0000 & 0,3725 & 0,5618 & 0,5736 \\
\hline Unid 19 & 0,7882 & 1,0000 & 0,3941 & 0,6065 & 0,7133 & 1,0000 & 0,3567 & 0,5379 & 0,5722 \\
\hline Unid 20 & 0,7144 & 0,9814 & 0,3665 & 0,5640 & 0,7152 & 0,9757 & 0,3697 & 0,5576 & 0,5608 \\
\hline
\end{tabular}

Fonte: elaborado pelos autores.

*normalizada

Duas unidades chamam atenção por apresentarem melhores posições no segundo semestre: UNID 13 e UNID 14, ambas melhorando 3 posições.

Todas as demais unidades praticamente mantiveram os valores da eficiência composta normalizada e as posições no ranking das unidades.

Pensando em categorizar as unidades por graus de eficiência, pode-se classificá-las como Unidade de Alta, Média ou Baixa Eficiência. Sugerindo o uso dos valores média e desvio padrão da eficiência composta normalizada nos dois semestres tem-se os valores de referência e a quantidade de unidades distribuídas em cada classificação, conforme apresentado na Tabela 2.

Tabela 2 Classificação das unidades por eficiência

\begin{tabular}{cccc} 
Classificação & $\begin{array}{c}\text { Valor da eficiência composta } \\
\text { normalizada (Média composta*) }\end{array}$ & $\begin{array}{c}\text { Número de } \\
\text { unidades }\end{array}$ & Unidades \\
\hline Alta Eficiência & Valor $\geq 0,9042$ & 3 & Unid 1 a 3 \\
Média Eficiência & $0,6318 \leq$ Valor $<0,9042$ & 13 & Unid 4 a 16 \\
Baixa Eficiência & Valor $<0,6318$ & 4 & Unid 17 a 20 \\
\hline
\end{tabular}

Fonte: elaborado pelos autores. 
Tais resultados podem ser utilizados para identificar as melhores práticas, servindo as três primeiras unidades como referência em termos de eficiência produtiva para as demais.

Na Análise Envoltória de Dados também são analisadas as influências exercidas por algumas unidades sobre as outras. A Tabela 3 apresenta as unidades de referência para as quatro unidades classificadas como unidades com baixa eficiência na análise anterior. Quanto mais próximo de 1 , mais forte é a influência. A identificação das unidades de referência pode ser utilizada como informação estratégica pelas unidades ineficientes, uma vez que práticas operacionais e projetos estratégicos podem ser compartilhados entre elas.

Tabela 3 Principais benchmarks das unidades com baixa eficiência

\begin{tabular}{cccccc} 
Unidades com & \multicolumn{5}{c}{ Unidades de referência } \\
baixa eficiência & \multicolumn{3}{c}{ Primeiro semestre } & \multicolumn{3}{c}{ Segundo semestre } \\
\hline \multirow{2}{*}{ Unid 20 } & Unid 2 & Unid 6 & Unid 1 & Unid 2 & Unid 6 \\
& 0,2452 & 0,1862 & 0,1579 & 0,2682 & 0,2007 \\
Unid 19 & Unid 1 & & & Unid 1 & \\
& 0,1203 & & & 0,1498 & \\
Unid 18 & Unid 2 & Unid 5 & Unid 1 & Unid 2 & \\
& 0,3926 & 0,1223 & 0,1152 & 0,4349 & \\
Unid 17 & Unid 10 & & & Unid 2 & Unid 1 \\
& 0,588 & & & 0,1197 & 0,1097 \\
\hline
\end{tabular}

Fonte: elaborado pelos autores.

Pela Tabela 3, percebe-se que a Unid 20 deve manter contato com as unidades Unid 2, Unid 6 e Unid 1 para conhecer suas realidades e compartilhar práticas. A Unid 19 deve "seguir" as boas práticas da Unid 1 e assim por diante.

A análise de benchmark sugere relacionamentos que podem ser fortalecidos na Universidade. No entanto torna-se necessário também analisar os alvos para as variáveis tratadas neste estudo de forma que as unidades com baixa eficiência identifiquem os pontos onde devem atuar e transformem-se em eficientes.

A Tabela 4 apresenta os valores para as quatro unidades classificadas como unidades com baixa eficiência anteriormente. A última coluna apresenta a variação percentual entre o valor atual e o alvo sugerido.

Tabela 4 Alvos e folgas das unidades com baixa eficiência

\begin{tabular}{|c|c|c|c|c|c|c|c|}
\hline & & Variável & Atual & Radial & Folga & Alvo & $\begin{array}{c}\text { VariaçãoAtual } \\
x \text { Alvo }\end{array}$ \\
\hline \multirow{4}{*}{ 옴 } & \multirow{4}{*}{ :블 } & Qde_cursos & 13 & 13 & 0 & 13 & $0 \%$ \\
\hline & & Custo_docente & $1.728 .735,00$ & $1.728 .735,00$ & $7.895,82$ & $1.720 .839,18$ & $-0,5 \%$ \\
\hline & & Cred_of & 33.109 & 33.109 & 0 & 33.109 & $0 \%$ \\
\hline & & outras_desp & $1.433 .232,00$ & $1.433 .232,00$ & 0,00 & $1.433 .232,00$ & $0 \%$ \\
\hline
\end{tabular}




\begin{tabular}{|c|c|c|c|c|c|c|c|}
\hline & & Qde alunos & 1.528 & 2.139 & 242 & 2.380 & $56 \%$ \\
\hline & & Cred_ocup & 20.992 & 29.382 & 0 & 29.382 & $40 \%$ \\
\hline & & Rec_cursos & $5.300 .787,00$ & 7.419.490,81 & 0,00 & $7.419 .490,81$ & $40 \%$ \\
\hline & \multirow{7}{*}{ 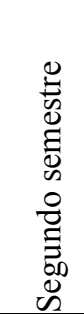 } & Qde_cursos & 14 & 14 & 2 & 12 & $-13 \%$ \\
\hline & & Custo_docente & $2.125 .799,00$ & $2.125 .799,00$ & 0,00 & $2.125 .799,00$ & $0,0 \%$ \\
\hline & & Cred_of & 32.058 & 32.058 & 0 & 32.058 & $0 \%$ \\
\hline & & outras_desp & $1.383 .278,00$ & $1.383 .278,00$ & $56.816,04$ & $1.326 .461,96$ & $-4 \%$ \\
\hline & & Qde_alunos & 1.458 & 2.039 & 195 & 2.233 & $53 \%$ \\
\hline & & Cred_ocup & 19.714 & 27.566 & 0 & 27.566 & $40 \%$ \\
\hline & & Rec_cursos & $5.094 .001,00$ & $7.122 .870,78$ & 0,00 & $7.122 .870,78$ & $40 \%$ \\
\hline \multirow{14}{*}{ 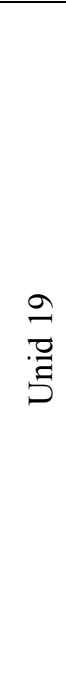 } & \multirow{7}{*}{ 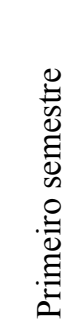 } & Qde_cursos & 4 & 4 & 2 & 2 & $-52 \%$ \\
\hline & & Custo_docente & $286.287,00$ & $286.287,00$ & 0,00 & $286.287,00$ & $0 \%$ \\
\hline & & Cred_of & 9.112 & 9.112 & 3.037 & 6.075 & $-33 \%$ \\
\hline & & outras_desp & $235.729,00$ & $235.729,00$ & 0,00 & $235.729,00$ & $0 \%$ \\
\hline & & Qde_alunos & 318 & 403 & 19 & 422 & $33 \%$ \\
\hline & & Cred_ocup & 4.064 & 5.156 & 0 & 5.156 & $27 \%$ \\
\hline & & Rec_cursos & $979.640,00$ & $1.242 .838,87$ & 0,00 & $1.242 .838,87$ & $27 \%$ \\
\hline & \multirow{7}{*}{ 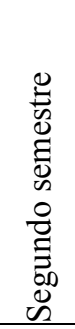 } & Qde_cursos & 4 & 4 & 2 & 2 & $-52 \%$ \\
\hline & & Custo_docente & $392.451,00$ & $392.451,00$ & 0,00 & $392.451,00$ & $0 \%$ \\
\hline & & Cred_of & 8.528 & 8.528 & 1.858 & 6.670 & $-22 \%$ \\
\hline & & outras_desp & $245.818,00$ & $245.818,00$ & 0,00 & $245.818,00$ & $0 \%$ \\
\hline & & Qde_alunos & 310 & 435 & 16 & 451 & $45 \%$ \\
\hline & & Cred_ocup & 3.924 & 5.501 & 0 & 5.501 & $40 \%$ \\
\hline & & Rec_cursos & $965.886,00$ & $1.354 .065,67$ & 0,00 & $1.354 .065,67$ & $40 \%$ \\
\hline \multirow{14}{*}{ 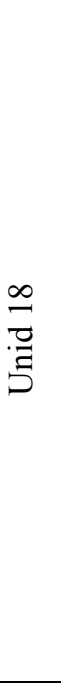 } & \multirow{7}{*}{ 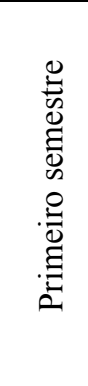 } & Qde_cursos & 15 & 15 & 0 & 15 & $0 \%$ \\
\hline & & Custo_docente & $1.646 .555,00$ & $1.646 .555,00$ & 0,00 & $1.646 .555,00$ & $0 \%$ \\
\hline & & Cred_of & 33.138 & 33.138 & 0 & 33.138 & $0 \%$ \\
\hline & & outras_desp & $1.337 .706,00$ & $1.337 .706,00$ & 0,00 & $1.337 .706,00$ & $0 \%$ \\
\hline & & Qde_alunos & 1.449 & 1.960 & 517 & 2.477 & $71 \%$ \\
\hline & & Cred_ocup & 22.914 & 30.992 & 0 & 30.992 & $35 \%$ \\
\hline & & Rec_cursos & $4.920 .567,00$ & $6.655 .309,34$ & 0,00 & $6.655 .309,34$ & $35 \%$ \\
\hline & \multirow{7}{*}{ 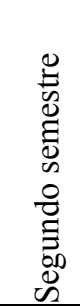 } & Qde_cursos & 16 & 16 & 3 & 13 & $-16 \%$ \\
\hline & & Custo_docente & $1.961 .066,00$ & $1.961 .066,00$ & 0,00 & $1.961 .066,00$ & $0 \%$ \\
\hline & & Cred_of & 30.206 & 30.206 & 0 & 30.206 & $0 \%$ \\
\hline & & outras_desp & $1.207 .387,00$ & $1.207 .387,00$ & $62.472,69$ & $1.144 .914,31$ & $-5 \%$ \\
\hline & & Qde_alunos & 1.340 & 1.799 & 445 & 2.244 & $67 \%$ \\
\hline & & Cred_ocup & 20.978 & 28.160 & 0 & 28.160 & $34 \%$ \\
\hline & & Rec_cursos & $4.457 .554,00$ & 5.983.538,54 & 0,00 & $5.983 .538,54$ & $34 \%$ \\
\hline \multirow{9}{*}{ 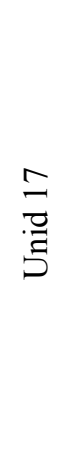 } & \multirow{7}{*}{ 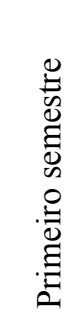 } & Qde_cursos & 8 & 8 & 0 & 8 & $0 \%$ \\
\hline & & Custo_docente & $611.966,00$ & $611.966,00$ & $65.870,29$ & $546.095,71$ & $-11 \%$ \\
\hline & & Cred_of & 12.440 & 12.440 & 894 & 11.546 & $-7 \%$ \\
\hline & & outras_desp & $320.228,00$ & $320.228,00$ & 0,00 & $320.228,00$ & $0 \%$ \\
\hline & & Qde_alunos & 519 & 602 & 0 & 602 & $16 \%$ \\
\hline & & Cred_ocup & 6.748 & 7.833 & 717 & 8.550 & $27 \%$ \\
\hline & & Rec_cursos & $1.316 .483,00$ & $1.528 .151,11$ & 0,00 & $1.528 .151,11$ & $16 \%$ \\
\hline & $\mathscr{d}$ & Qde_cursos & 7 & 7 & 3 & 4 & $-41 \%$ \\
\hline & 응 맹 & Custo_docente & $798.406,00$ & $798.406,00$ & $293.056,87$ & $505.349,13$ & $-37 \%$ \\
\hline
\end{tabular}




\begin{tabular}{lccccc}
\hline Cred_of & 12.024 & 12.024 & 2.377 & 9.647 & $-20 \%$ \\
outras_desp & $321.725,00$ & $321.725,00$ & 0,00 & $321.725,00$ & $0 \%$ \\
Qde_alunos & 517 & 694 & 38 & 732 & $42 \%$ \\
Cred_ocup & 6.616 & 8.879 & 0 & 8.879 & $34 \%$ \\
Rec_cursos & $1.303 .046,00$ & $1.748 .808,60$ & 0,00 & $1.748 .808,60$ & $34 \%$ \\
\hline
\end{tabular}

Fonte: elaborado pelos autores.

Percebe-se que, de forma geral, a quantidade de alunos é a variável que apresentou os maiores valores na coluna variação. Esta informação somente confirma o que já é de conhecimento na Universidade, que algumas unidades sofrem por falta de alunos.

Destacam-se, entretanto, outras informações: as unidades Unid 20 e Unid 18 estão praticamente ajustadas quanto às variáveis utilizadas como inputs na análise, que são a quantidade de cursos, o custo docente, quantidade de créditos oferecidos e outras despesas, o que sugere que sua estrutura está adequada. Desta forma, uma das ações possíveis é intensificar ações que agreguem alunos e que façam os alunos adquirirem mais créditos. Como consequência, estas ações resultariam em aumento das receitas dos cursos.

Por outro lado, as unidades Unid 19 e Unid 17 devem rever sua estrutura, pois, pela análise, as variáveis de entrada deveriam ser drasticamente reduzidas. As ações necessárias para estas unidades exigem adequação da estrutura. As sugestões para estas ações podem ser obtidas através do uso de benchmark das unidades Unid 1, Unid 2 e Unid 10 (conforme Tabela 3).

Ampliando-se a análise, buscou-se validar os resultados obtidos com o modelo DEA através de seu comparativo com análises tradicionais já utilizadas na Instituição. Foi realizado o cruzamento entre o percentual da margem de contribuição sobre a receita de cada unidade com o valor da eficiência obtido na análise DEA, conforme apresentado no Gráfico 1. As unidades representadas no Gráfico 1 também foram divididas pela sua característica predominante (Sociais Aplicadas, Núcleos Universitários, Tecnologias e Licenciaturas). 


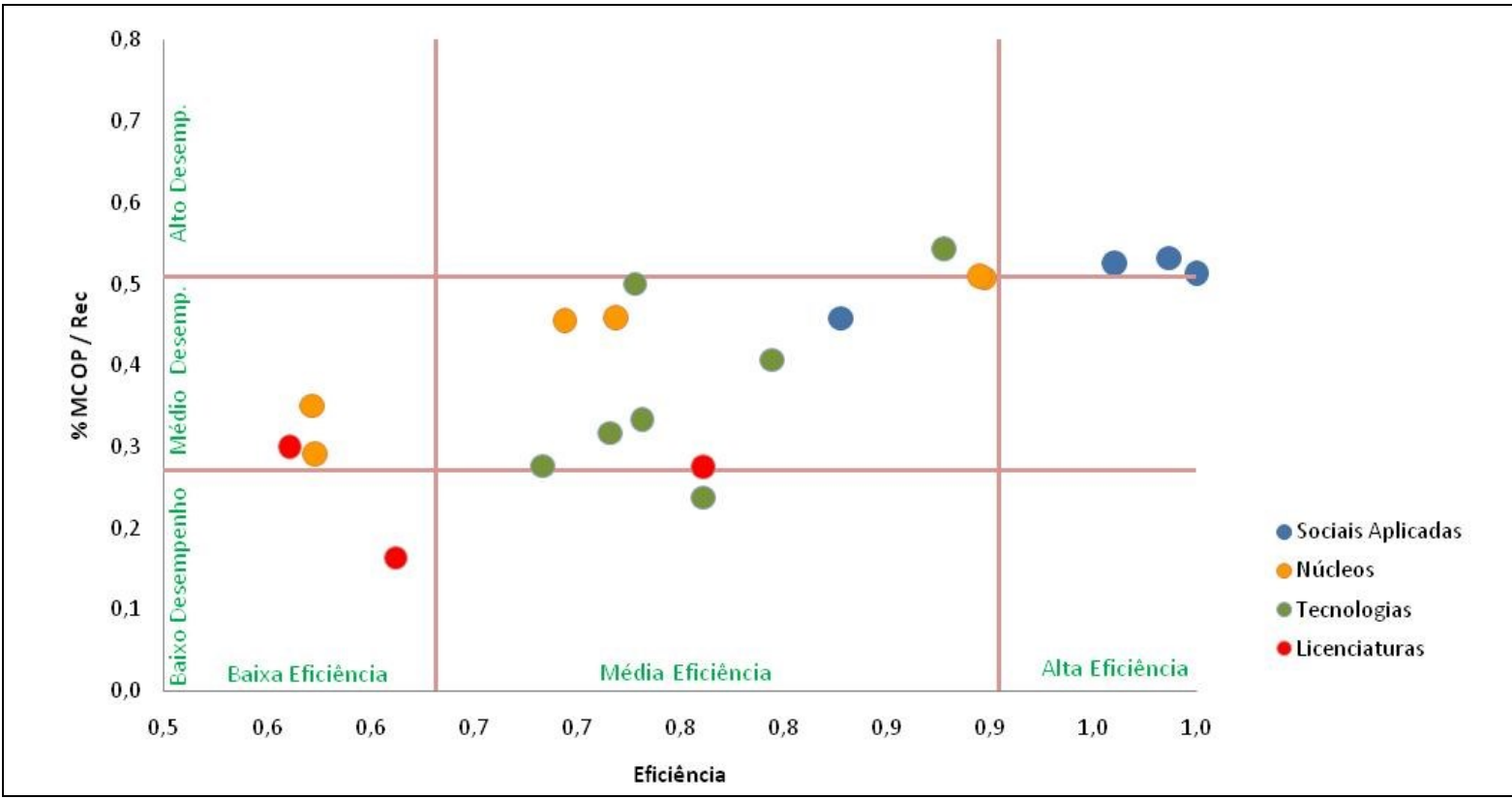

Gráfico 1 Relação MC/Receita e Eficiência Fonte: elaborado pelos autores.

Observando-se o Gráfico 1, percebe-se que as duas análises são coerentes entre si. Unidades classificadas como sendo de Baixa Eficiência também apresentam Desempenhos baixos, de forma geral.

\section{CONCLUSÕES}

A avaliação correta do emprego e utilização de recursos nas Universidades se tornou imprescindível, principalmente nos últimos anos quando a concorrência entre as IES se intensificou. Entre as várias formas de avaliação do desempenho de unidades acadêmicas, uma das possibilidades está no uso da Análise Envoltória de Dados, que compara unidades de decisão que possuem as mesmas características em função das variáveis de entrada e saída.

Neste estudo foram analisadas 20 unidades acadêmicas da Universidade de Caxias do Sul, utilizando-se os dados de dois semestres de 2012.

Com os resultados obtidos na análise de eficiência, as unidades foram classificadas em três grupos, resultando quatro unidades com classificação de unidade com baixa eficiência. Para estas unidades procedeu-se análise dos benchmarks e de alvos e folgas, indicando onde devem ser tomadas medidas de forma a melhorar a eficiência destas unidades e quais as outras unidades que poderiam servir de benchmark por possuírem características em comum. 
É importante destacar que os resultados sugeriram para estas unidades a necessidade de aumento de alunos. Tal informação não é novidade, porém é de difícil solução, uma vez que algumas unidades sofrem mais por influência de variáveis externas à própria instituição, que afetam não somente esta universidade, mas as mesmas áreas de ensino em todo o Brasil.

Embora tenham sido escolhidos indicadores relacionados ao ensino de graduação destas unidades acadêmicas, sugere-se estender as análises com a exclusão e inclusão de indicadores, que ampliem a análise para aspectos de desempenho dos cursos, sejam eles desempenhos financeiros, mas também de qualidade e satisfação dos alunos. A escolha depende dos objetivos estratégicos e das necessidades de cada unidade, mas permitirá identificar os pontos onde cada unidade pode evoluir.

Finaliza-se este trabalho afirmando que a utilização desta ferramenta pode auxiliar os gestores na avaliação das unidades de ensino, permitindo uma melhor gestão dos recursos consumidos e resultados obtidos. Bem como, fomentar a importância de um maior aculturamento na utilização de ferramentas para avaliação de desempenho em instituições de ensino superior.

\section{REFERÊNCIAS}

ANGULO MEZA, L; BIONDI NETO, L.; SOARES DE MELLO, J.C.C.B.; GOMES, E. G. ISYDS- Integrated System for Decision Support (SIAD - Sistema Integrado de Apoio a Decisão): a software package for data envelopment analysis model. Pesquisa Operacional, v.25, n.3, p 493-503. 2005.

ANTOUN NETTO, S. O. ; LINS, M. P. E. Análise envoltória de dados (DEA) aplicada na determinação de indicadores de desempenho das capitais e do distrito federal brasileiros na área de saúde. In: XVI CLAIO (Congreso Latino-Iberoamericano de Investigación Operativa / XLIV SBPO (Simpósio Brasileiro de Pesquisa Operacional), Rio de Janeiro, 2012.

BANKER, R.D.; CHARNES, A.; COOPER, W.W. Some models for estimating technical scale inefficiencies in data envelopment analysis. Management Science, v. 30, n. 9, p. 10781092, 1984.

BELLONI, I. Uma Metodologia de Avaliação da Eficiência Produtiva de Universidades Federais Brasileiras. Tese de Doutorado, UFSC, 2000.

BERLINER, C. \& BRIMSON, J. A.; Gerenciamento de custos em indústrias avançadas: base conceitual CAM-I. T. A. QUEIROZ, EDITOR, LTDA. São Paulo, 1992.

CALVO, M. C. M. Hospitais públicos e privados no Sistema Único de Saúde do Brasil: o mito da eficiência privada no estado de Mato Grasso em 1998. 223 f. 2002. Tese (Doutorado 
em Engenharia de Produção) - Universidade Federal de Santa Catarina, Florianópolis/SC, 2002.

CAMPOS, V. F. TQC: Controle da Qualidade Total (no estilo japonês). 3. ed. Belo Horizonte: Fundação Christiano Ottoni, Escola de Engenharia da UFMG, 1992. 220p.

CASADO, Frank Leonardo; SILUK, Julio Cezar. Avaliação da eficiência de unidades universitárias de uma instituição de ensino superior. In: XLIII Simpósio Brasileiro de Pesquisa Operacional (XLIII SBPO 2011), 2011, Ubatuba, SP

CASADO, Frank Leonardo; SOUZA, Adriano Mendonça. Análise envoltória de dados: conceitos, metodologia e estudo da arte na educação superior. Revista Sociais e Humanas, Santa Maria, v. 1, p. 1-154, Jun. 2007.

CAVALCANTE, S. M. e ANDRIOLA, W. Avaliação da eficiência dos cursos de graduação da Universidade Federal do Ceará (UFC) através da Análise Envoltória de Dados (DEA). Revista Iberoamericana de Evaluación Educativa. V. 5, n. 3, p. 291-314, 2012.

CHARNES, A. et al. Data Envelopment Analysis: theory, methodology and applications. USA Khuwer Academic Publishers, 1994.

CHARNES, A.; COOPER, W. W.; RHODES, E. Measuring the efficiency of decisionmaking units. European Journal of Operational Research, v. 2, p.429-444, 1978.

COSTA, Edward Martins. Mensuração da eficiência produtiva das instituições Federais De Ensino Superior - IFES, XV Prêmio Tesouro Nacional, Trabalho Completo. 2010.

ENCINAS, R. Oportunidades de aplicação da análise envoltória de dados em auditorias operacionais do Tribunal de Contas da União. 2010. Disponível em:

$<$ http://portal2.tcu.gov.br/portal/pls/portal/docs/2054734.PDF $>$. Acesso em: 09 de maio 2013.

ENTANI, T., MAEDA Y., TANAKA, H. Dual Models of interval DEA and its extensions to interval data. European Journal of Operation Research, 136, 32-45, 2002.

FAÇANHA, L. O.; MARINHO, A. Instituições federais de ensino superior: Modelos de financiamento e o incentivo à eficiência. Revista Brasileira de Economia, 53(3), p. 357-386, 2001 .

FARIA, F. P., JANNUZZI, P. M., SILVA, S. J. Eficiência dos gastos municipais em saúde e educação: uma investigação através da análise envoltória no estado do Rio de Janeiro. Revista de Administração Pública, v. 42, n. 1, p. 155-177, jan./fev. 2008.

FRIED, H.; LOVELL, C.A.K. e SCHMIDT, S.S. The measurement of productive efficiency. New York: Oxford University, 1993.

GOLDRATT, E. M.; FOX, R. E. A corrida pela vantagem competitiva. São Paulo, Educator. 1992. 
GOMES, Eliane Gonçalves; SOUZA, Geraldo da Silva e; LIMA, Suzana Maria Valle; FONSECA, Carlos Eduardo Lazarini. Alocação de bolsas de iniciação científica às unidades da EMBRAPA com modelos de análise envoltória de dados com ganhos de soma zero. ENGEVISTA, v. 9, n. 1, p. 14-21, junho 2007.

HAMMER, M.; These vendeadly sins of performance measurement and how to avoid them. MIT Sloan Management Review. Cambridge, v. 48, n. 3, p. 18-28, primavera 2007.

INSTITUTO NACIONAL DE ESTUDOS E PESQUISAS EDUCACIONAIS ANÍSIO TEIXEIRA. Censo da educação superior: 2010 - resumo técnico. - Brasília: Instituto Nacional de Estudos e Pesquisas Educacionais Anísio Teixeira, 2012. Disponível em:http://portal.mec.gov.br/index.php?option=com_content\&view $=$ article\&id=16179. Consultado em 21.11.2012.

KAPLAN, Robert S. e NORTON, David P. Organização orientada para a estratégia: como as empresas que adotam o Balanced Scorecard prosperam no novo ambiente de negócios. Rio de Janeiro: Campus, 2001.

KYJ, L. e PARKER, R. J. Antecedents of Budget Participation: Leadership Style, Information Asymmetry, and Evaluative Use of Budget. ABACUS, v. 44, n.. 4, p. 423-442, 2008.

LETA, F. R.; SOARES de MELLO, J.C.C.B.; GOMES, E.G.; ÂNGULO MEZA, L. Métodos de melhora de ordenação em DEA aplicados à avaliação estática de tornos mecânicos. Investigação Operacional, Lisboa, v. 25, n. 2, p. 229-242, 2005.

LINS, M. E. ET AL. Análise Envoltória de Dados (DEA) para avaliação de hospitais universitários brasileiros. Ciência \& Saúde Coletiva, v.12, n.4, p. 985-998, 2007.

LINS, M. P. E.; ALMEIDA, B. F. de; BARTHOLO JR, R. Avaliação de desempenho na pósgraduação utilizando a Análise Envoltória de Dados: o caso da Engenharia de Produção. Revista Brasileira de Pós-Graduação. n. 1. p. 41-56. 2004.

LOBO, M. S. C. Aplicação da análise envoltória de dados (DEA) para apoio às políticas públicas de saúde: o caso dos hospitais de ensino. 2010. 228f. Tese (Doutorado em Engenharia de Produção) - Universidade Federal do Rio de Janeiro, Rio de Janeiro/RJ, 2010.

MACEDO, M. A. S.; SANTOS R. M.; SILVA F. F. Avaliação de desempenho organizacional: utilizando análise envoltória de dados (DEA) em informações financeiras e não financeiras. In: Encontro Nacional de Engenharia de Produção, 24, 2004. Florianópolis. Anais. Florianópolis: UFSC, 2004. 1 CD-ROM.

MAÑAS, A. V. Gestão de tecnologia e inovação. São Paulo: Érica, 1993.

MARINHO, A. Avaliação da eficiência técnica nos serviços de saúde nos municípios do Estado do Rio de Janeiro. Revista Brasileira de Economia, Rio de Janeiro, v. 57, n. 3, Set. 2003. 
NOVAES, L. F. L. Envoltória Sob Dupla ótica aplicada na avaliação imobiliária em ambiente do sistema de informação geográfica. Tese de Doutorado, Programa de Engenharia de Produção. UFRJ, Rio de Janeiro, 2002.

OLIVEIRA, C. E. M.; TURRIONI, J. B. Medidas de desempenho em instituições federais de ensino superior - um estudo sobre o Balanced Scorecard. In: ENCONTRO DE ENGENHARIA DE PRODUÇÃO DA UFRJ - PROFUNDÃO, 10, 2006, Rio de Janeiro. Anais... 2006.

OLIVEIRA, N. G. A.; ARAUJO, M. I. S.; BATISTA, M. G.; DERZI, E. C. M.; YAMAGUCHI, H. K. L. Análise Envoltória de Dados: um levantamento bibliográfico dos modelos DEA aplicados no setor educacional no Brasil, período de 1999 a 2009. In: XXXI Encontro Nacional de Engenharia de Produção, 2011.

PANEPUCCI, G. T. M. Avaliação de desempenho dos departamentos acadêmicos da UFScar utilizando a análise de envoltória de dados-AED. Dissertação de Mestrado. Universidade Federal de São Carlos, UFSCAR, Brasil, 2003.

SAATY, T. L.; Método de Análise Hierárquica. São Paulo. Editora Makron, 1991.

SMITH, M.; Performance Management Methodology. Business Credit. Columbia, v.107, n.10, p.54-5,nov.-dez. 2005.

SOARES DE MELLO, J. C. C. B.; ÂNGULO MEZA, L.; GOMES, E. G.; BIONDI NETO, L. Curso de Análise de Envoltória de Dados. XXXVII SBPO - Simpósio Brasileiro de Pesquisa Operacional, 2005, Gramado. Anais do XXXVII SBPO, 2005.

SOARES DE MELLO, M. H. C.; QUINTELLA, H. L. M. M.; SOARES DE MELLO, J. C. C. B. Avaliação do desempenho de alunos considerando classificações obtidas e opiniões dos docentes. Investigação Operacional, v. 24, 2004. 